International Journal of Robotic Computing

Vol. 1, No. 2 (2019)

(C) KS Press, Institute for Semantic Computing Foundation

DOI: $10.35708 / \mathrm{RC} 1868-126254$

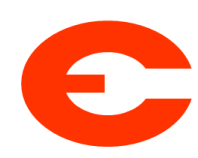

\title{
Pouring from Deformable Containers Based on Tactile Information Using a Dual-Arm Robot
}

\author{
Yuto Tsuchiya, Takuya Kiyokawa, Gustavo Alfonso Garcia Ricardez, \\ Jun Takamatsu, and Tsukasa Ogasawara \\ Nara Institute of Science and Technology (NAIST) \\ 8916-5 Takayama, Ikoma, Nara, 630-0192, Japan \\ \{tsuchiya.yuto.tu5, kiyokawa.takuya.kj5, garcia-g, j-taka, \\ ogasawar\}@is.naist.jp
}

Received $(11 / 01 / 2018)$

Revised (06/15/2019)

Accepted (07/20/2019)

\begin{abstract}
In this paper, we consider household robots that pour various contents from deformable containers. Such pouring is often seen in cooking and refilling. To achieve this kind of pouring, we reduce the deformation of the container during pouring and thus carefully design the grasping strategy: the palm of one hand supports the deformable container from the bottom and the other hand pulls up the container from the top. We apply the proposed system to pouring four different kinds of contents: breakfast cereal, coffee beans, flour, and rice. The experiment verifies that the proposed system successfully pours the four contents. To evaluate the system quantitatively, we measure 1) the deformation of the container using a motion capture system and 2) the success rate of pouring. We verify that the dual-arm pouring reduced the deformation by $66 \%$ compared to a single-arm motion and that the success rate is greater than $90 \%$.
\end{abstract}

Keywords: Deformable object; Pouring motion; Tactile information; Dual-arm robot.

\section{Introduction}

There are many packed objects in a household, some of which are commercial products acquired at supermarkets or convenience stores. To deliver these products containing diverse ingredients to the points of sale, the products are carefully packed to ensure the integrity of their contents. Once we have the products at home, we need to unpack them. Pouring is the representative method to unpack 
the contents, such as grains, liquids, and powder. Thus, pouring motion is often seen in cooking and when refilling a detergent to a reusable container [10, for example.

Deformable containers are commonly used in our daily life. Figure 1 shows the classification of the products that we collected with respect to types of contents and containers. There are four types of contents (liquids, paste, grains, and powder) and three types of containers (retort pouch, plastic bag, and paper bag).

One of the methods to make robots pour contents from containers is to create physical models of the target objects and to plan the motion by using such models [17]19]3. Compared to a rigid container, the state of a deformable container is represented as a large (maybe infinite) number of parameters. Thus, it is difficult to model the container. Moreover, contents also deform during a pouring motion. This deformation complicates the realization of the robot pouring.

In contrast to the model-based method, we adopt a strategy to reduce the deformation of a container. We think that the deformation leads to unexpected effects. We point out two important aspects to reduce the deformation. First, the products are designed to be easily delivered. The products can keep their forms in an upright orientation. In other words, tilting the container to pour the contents leads to deforming the container and its contents. Second, there is the dilemma about what force should be applied to support the container since the higher the applied force is, the higher the possibility of deformation. The key to reduce the deformation is to appropriately decide the contact areas and forces when tilting the container.

In this paper, we propose a robot system to pour various contents from deformable containers. We assume that the robot has two arms and force sensors at the fingertips. When tilting the container, the robot supports the container by 1) lifting up the container from the top using one hand, and 2) half-enclosing the container from the bottom using the other hand. In order to reduce the deformation, the hands apply the minimum force to keep the grasp.

Through experiments, we verify the effectiveness of the proposed method using two indices. One index is the amount of the deformation during pouring motion. Another index is the success rate of pouring. We also investigate the difference in the amount of pouring in twenty trials while carefully initializing the state of the container to make it as similar as possible across all trials.

This paper is an extension of our IRC 2019 paper [18. In the present work, we add three new materials.

1. Conducting experiments in more realistic scenarios: We add the experiments to evaluate the proposed system from various points of views. The original paper presented experiments where the robot pours four types of contents from a plastic bag (Section 5.1). This paper additionally evaluates the system in a more realistic scenario (Section 5.2), where we directly use four actual commercial products. We verified that the system could pour all of them by adjusting grasping forces and grasping positions. 


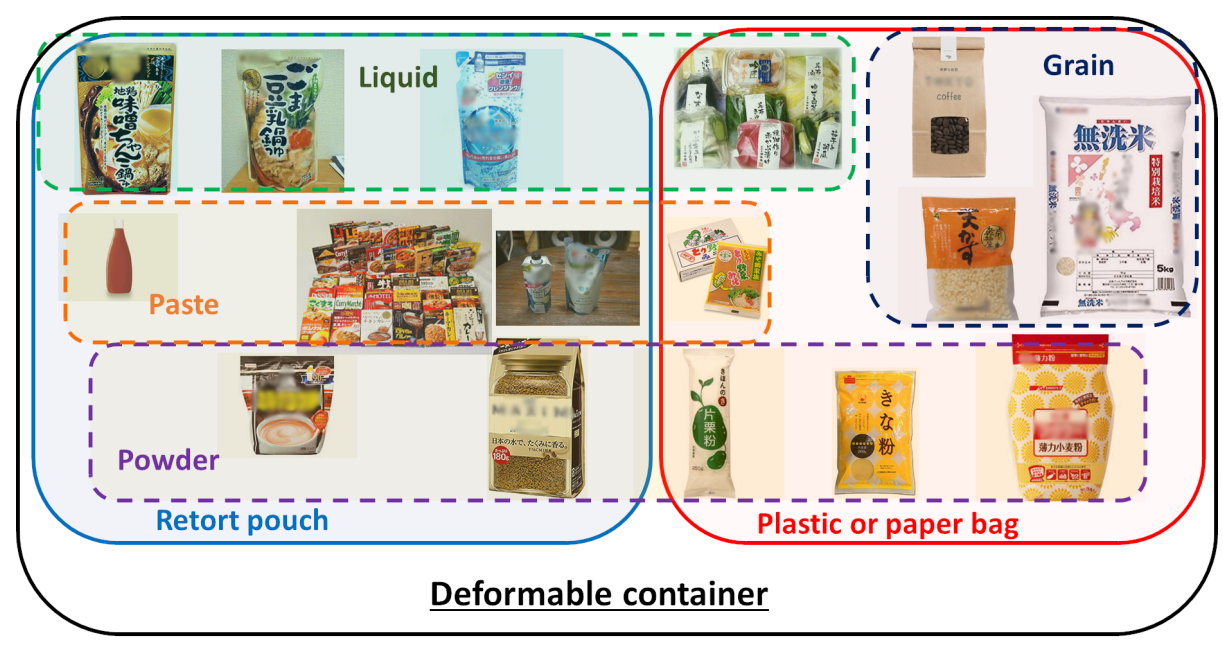

Fig. 1. Commercial products with deformable containers at home

2. Evaluating the proposed system by new indices: In the present work, we evaluate the system using two indices. One index is the success rate of pouring. This is not included in the original work of IRC 2019. The robot poured each type of contents twenty times. The success rate was greater than $90 \%$. The other index is the amount of the deformation of a container during pouring motions. This is used in the original work. We also investigate the amount of pouring (Section 5.5). In our proposed system, the arms move in a feed-forward manner and the grasping forces are controlled in a feedback manner. We confirmed that the amount of pouring deviates at some level.

3. Adding the information useful for the implementation: In this paper, we described the method for generating the arm movements, the specification (e.g., the reachability of the arms of our robot described in Section 4.1).

\section{Related work}

As the target field of robots has been changing to home environments, many research methods aimed at grasping, manipulation, and sensing of deformable objects 813]. Many household tasks are involved in deformable object manipulation such as folding clothes [1,24] and handling foods in a kitchen [26]4]. In the research on robotic pouring, the target object has also been changing from rigid objects to deformable objects, in association with that main application field has been changed from industrial [17]19] to home environments [2315]. This paper pursues a robot pouring from a deformable container.

To realize a robot pouring equivalent to human, Learning-from-Demonstration (LfD) tends to be used, since it looks difficult to formulate the skillful low-level motion in pouring. In fact, the motion planning by formulating physical fluid model would be effective for precise pouring, but the planning method was evaluated only in simulation environments [9]. 
Yamaguchi et al. [22 21/23] employed LfD to derive a model of high-level skills of pouring such as pouring by tipping, shaking, and tapping. They developed a general pouring model to choose the proper skills considering situations such as material types, container shapes, initial poses of containers, and target amounts. Tamosiunaite et al. [16] proposed to use the Dynamic Movement Primitives (DMP) to represent the observed pouring motion for various target positions. Rozo et al. $[12$ measured the human demonstrations and used a parametric hidden markov model to relate force sensing with appropriate actions. In these research methods, the target objects to pour are a glass of water and a cup with granular, and the container is rigid or a viscoelastic object.

To pour from deformable containers is more challenging because they are easy to deform during pouring motion. Rodríguez et al. [11 tested pouring some contents from a deformable container using a single robot arm. By controlling the movement of 5 fingers equipped with an individual tactile sensor, their robot system stabilizes the contact configuration at the grasping points of a deformable object. However, single-arm pouring is prone to failure, in fact, in case of not applying their control method based on tactile properties at the 5 fingertips, the single robot arm dropped the container over in the experiment. Thus, in this paper, without such the control method of 5 finger movement, we propose the pouring from deformable containers by grasping target objects using dual arm and considering the macro movement of human-like dual arm motion to prevent the deformation and slippage.

Contents to pour is ranging from solid to liquid, and the dynamics of the contents changes. The sensor feedback in the pouring relieves modeling the dynamics. A vision system is taken advantage of for a robot to pour a specific amount of contents. Yamaguchi et al. [20] proposed the stereo vision system to obtain 3D flow points of the poured contents. Schenck et al. [14]15|7] proposed the method to estimate the amount of poured liquid using convolutional neural networks. As another usage, Guevara et al. [5] proposed to use a vision system to avoid spilling the contents by modifying the parameters of pouring motion. Since it is very challenging to do construct a robot system to pour various contents from a deformable container, in this paper, we concentrate on the achievement of a robot pouring. The visual feedback is future work.

\section{Proposed Robot System to Pour from a Deformable Container}

\subsection{Strategy for Pouring from a Deformable Container}

There are several phenomena leading to the deformation of a deformable container during pouring motion. First, by tilting the deformable container for pouring, one side of the container (referred to as a going-down side) is moved downwards (toward the recipient) and the contents move to the spout. The flow of the contents deforms the container. Second, the other side of container (referred to as a going-up side) is moved upwards, is not filled with the contents and loses rigidity. Thus, the container is also significantly deformed. Figure 2 shows the 


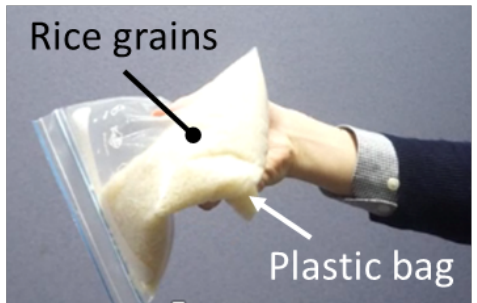

Fig. 2. An example of the shape deformation

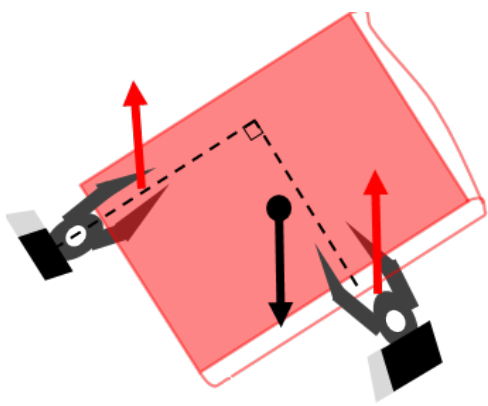

(a) Geometric relationship of two hands

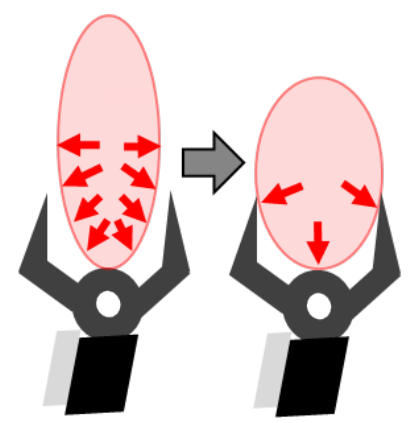

(b) The robot hand limits the expansion of the going-down side of the deformable container

Fig. 3. Dual-arm grasping avoiding the deformation

example of the deformation. If we pour the contents using a single arm, the container deforms significantly.

Considering these phenomena, we reduce the deformation using two approaches. First, the robot hand supports the going-down side of the deformable container and geometrically limits the expansion of the container. Second, by lifting up the going-up side of the deformable container, the bulging of the side is reduced. Using these two approaches, the robot restricts the deformation of the container.

Keeping in mind this strategy, we observed a human pouring motion. First, the human pours by holding both sides of a deformable container. Second, the human grasps the container with wider touching areas. As a result, the pressure is reduced while the force is maintained. Third, the human pours the contents by rotating the container while keeping the geometric relationship between her two arms.

To summarize the strategy, we first use a dual-arm robot with two hands, and, second, we mount force sensors on the robot hands. By measuring the grasping force exerted on the deformable container, the robot can achieve a good grasp with minimal deformation.

\subsection{Pouring using Two Arms}

Figure 3 shows the grasping using two hands. The robot grasps the going-down side of the container with one hand (referred to as the supporting hand) and the 


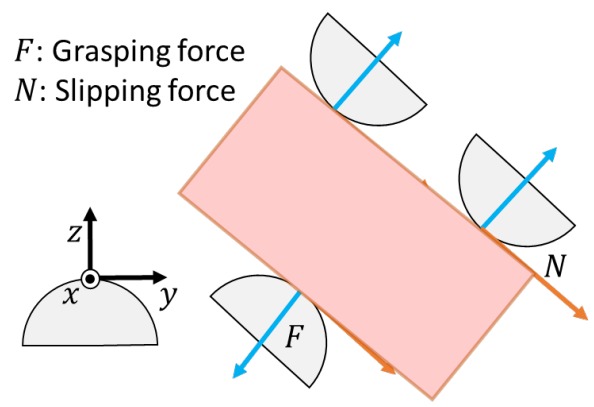

Fig. 4. Fingertip forces at contact points

bottom (near the going-up side) of the container with the other hand (referred to as the lifting hand). The former supports the weight of the container and the latter reduces the bulging of the side. Since both hands encompass wider areas, the pressure is reduced compared with only using the fingertips. Furthermore, as the contact area increases, the robot can restrain the shape change of the container.

In this paper, a predetermined pouring motion was performed to rotate the container while keeping constant the relative position and orientation of both hands. We define four coordinate systems: the coordinate systems of the robot $\Sigma_{r}$, the left arm $\Sigma_{l a}$, the right arm $\Sigma_{r a}$, and the rotation center $\Sigma_{c}$. The origin of the coordinate system $\Sigma_{c}$ is located at the rotation center, and the x-axis is set to align the rotation axis.

Let us consider the case of the left arm. The case of the right arm is the same. Let $\theta$ be the rotation angle from the upright stance. The configuration $M_{l a}^{r}$ of a left arm in $\Sigma_{r}$ satisfies Equation (1).

$$
M_{l a}^{r}(\theta)=M_{l a}^{c} M_{r o t}(\theta) M_{c}^{r},
$$

where $M_{l a}^{c}$ and $M_{c}^{r}$ represent the left-arm configuration in $\Sigma_{c}$ and the configuration of the center in $\Sigma_{r}$, and

$$
M_{\text {rot }}(\theta)=\left(\begin{array}{cccc}
1 & 0 & 0 & 0 \\
0 & \cos \theta & -\sin \theta & 0 \\
0 & \sin \theta & \cos \theta & 0 \\
0 & 0 & 0 & 1
\end{array}\right) .
$$

If the rotation center is given, $M_{c}^{r}$ is obtained, and $M_{l a}^{c}$ is obtained from the grasping position of the container. The arm configuration is obtained by solving its kinematics.

\subsection{Avoiding Deformation using Tactile Information}

Using a force sensor, the robot can control the grasping force exerted on the container. In this paper, we used a 3-finger gripper with 3D force sensors on each fingertip. Figure 4 shows the interaction between the container and the fingers. We focus on two types of forces: normal force $F$ and tangential force $N$. 


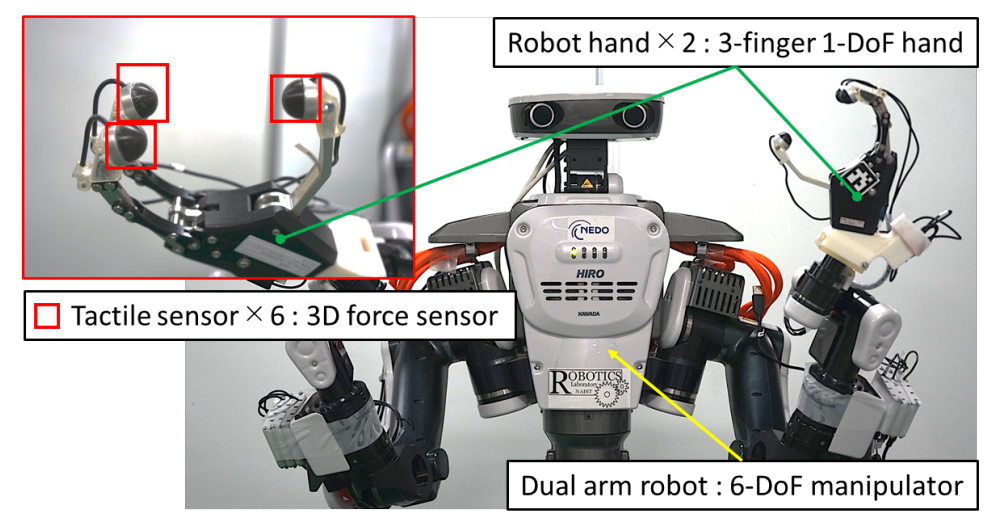

Fig. 5. The structure of our robot system

Generally, closing a hand increases the normal force and the maximum of the tangential force is formulated as the product of friction coefficient by the normal force.

As the normal force becomes smaller, the possibility of dropping the container is increased. On the other hand, as the normal force becomes bigger, the possibility of the deformation is increased. Thus, we estimate the suitable normal force experimentally and control the hand to make the measured value converge to the target value. For example, if the measured value is larger than the target value, the hand is opened.

Sudden change of the tangential force is caused by slipping between hand and container. The slipping leads to dropping the container. If a sudden change is detected, we close the hand to recover the tangential force to the original value.

\section{Implementation}

\subsection{Hardware Configuration}

Figure 5 shows the hardware configuration used in this paper. The dual-arm robot is a HIRO-NX manufactured by Kawada Robotics. Each arm has six degrees-of-freedom (DOF). The robotic hand at the end of the arm is a $T R X-S$ hand manufactured by THK Co., Ltd. This hand has three fingers to be able to hold an object and is underactuated with one DOF. In each fingertip, we attach the $3 \mathrm{D}$ force sensor $O M D-20-S E-40 N$ manufactured by Optoforce. The sensor can measure the force along the $\mathrm{x}^{-}, \mathrm{y}^{-}$, and $\mathrm{z}$-axes with high resolution: $2 \mathrm{mN}$ in the $\mathrm{x}$ - and $\mathrm{y}$-axes and $2.5 \mathrm{mN}$ in the $\mathrm{z}$-axis. The high resolution is advantageous to control the force of grasps.

Figure 6 shows the reachability of HIRO-NX's arms. In Figure 6(a) and Figure 6(b) the blue and red areas shows the reachable area of the right and left arms, respectively. In Figure 6(c) the green area shows the the reachable areas of both arms in the side view. We set the rotation center experimentally as the robot rotates the container until 120 degrees. 


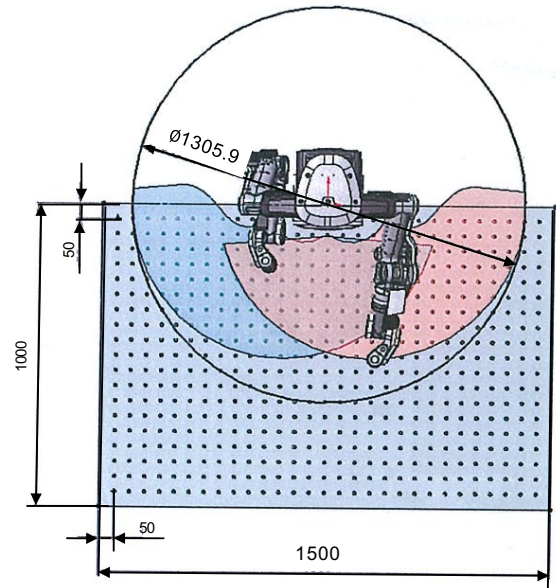

(a) Top view

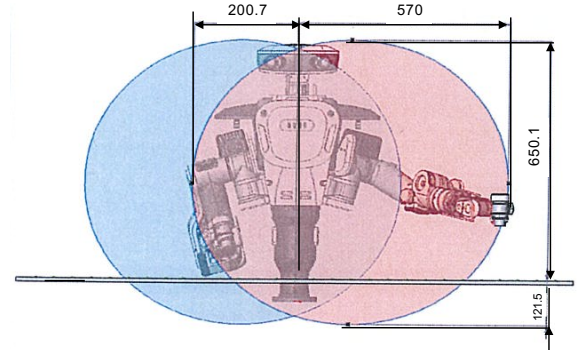

(b) Front view

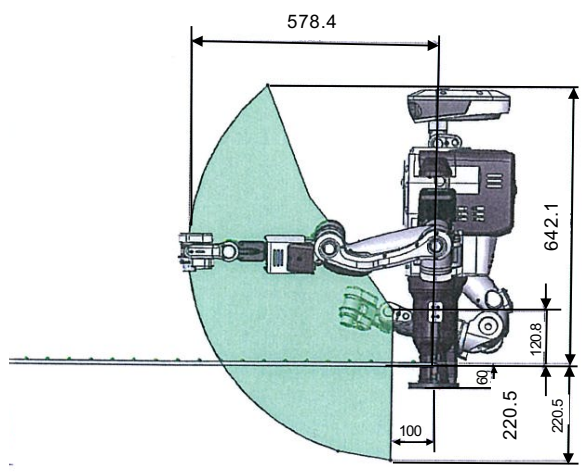

(c) Side view

Fig. 6. The movable range of both arms for HIRO-NX

\subsection{Software Configuration}

We implemented the software on ROS Kinetic Kame that works on Ubuntu 16.04 LTS. ROS (Robot Operating System) is a middleware for robot systems, which provides communication among nodes (e.g., robot controller, sensor, and gripper controller).

In our implementation, the robot motion is controlled independently from the gripper and the force sensor. The gripper changes the angles to adjust the force sensor's output to the pre-defined target value. We set the initial and the final angle of the rotation, $\theta$ in Equation (1). The trajectory of the rotation angle is obtained by linear interpolation.

\subsection{Control of Grasping Forces}

The grasping force is adjusted using the velocity-type PID control. In the velocitytype PID control, we calculate the difference of the control input at each step and avoid infinite number of sums for the numerical integration. 


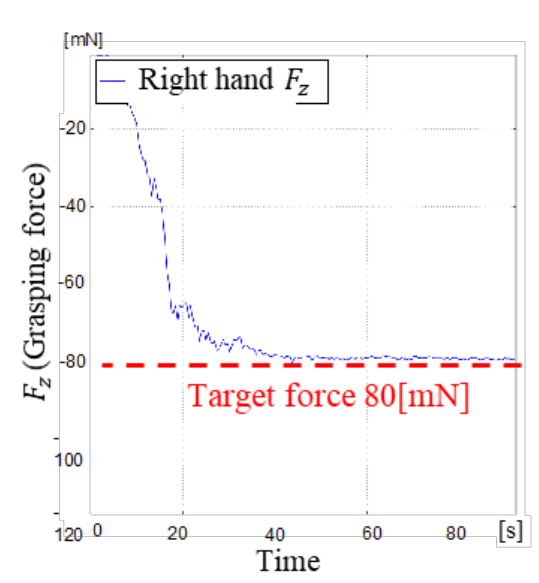

(a) Right arm

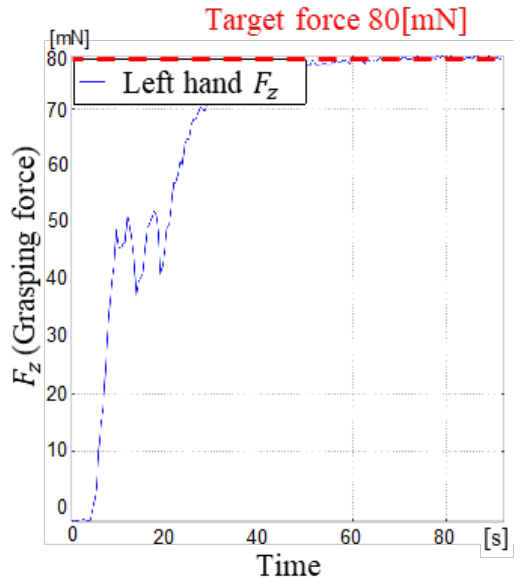

(b) Left arm

Fig. 7. Adjustment of grasping force by PID control

The input pulse for closing the TRX-S hand $u_{n}$ at time $n$ is calculated by Equations (2).

$$
u_{n}=u_{n-1}+\Delta u_{n o r m, n}+\Delta u_{t a n, n},
$$

where $\Delta u_{n o r m, n}$ and $\Delta u_{t a n, n}$ are the control values related to the normal force and the tangential force, respectively.

The control value $\Delta u_{n o r m, n}$ of the normal force is calculated by Equation (3).

$$
\begin{aligned}
\Delta u_{n o r m, n} & =K_{p}\left(e_{n}-e_{n-1}\right)+K_{i} e_{n}+K_{d}\left(e_{n}-2 e_{n-1}+e_{n-2}\right), \\
e_{n} & =F_{n o r m, n}-F_{n o r m, d}
\end{aligned}
$$

where $F_{n o r m, n}$ and $F_{n o r m, d}$ are the measured normal force at time $n$ and the target force, $K_{p}$ is the proportional gain, $K_{i}$ is the integral gain, and $K_{d}$ is the derivative gain. Furthermore, we limit the integral value of error by designating an upper limit and a lower limit, which improves the stability of the control.

The control value $\Delta u_{t a n, n}$ of the tangential force is calculated in the same way as Equation (3). As described earlier, when slippage happens, the tangential force is reduced. Then, the controller tries to stop the slippage by closing the hand. The target value is decided from the value just before slipping.

Figures $7(\mathrm{~b})$ and $7(\mathrm{a})$ show the target value of $F_{\text {norm }}$ using the above PID control on the right hand and the left hand when the robot pours breakfast cereal from a thin aluminium container. We set $F_{n o r m, d}$ to $80 \mathrm{mN}$ in both hands. The controller successfully adjusts the normal force.

\subsection{Procedure to Execute Pouring Motion}

The flow of the execution is as follows:

1. Move both arms to the initial positions (as shown in Figure 3(a). 
Table 1. Grasping force for contents

\begin{tabular}{lr}
\hline Material & Force $[\mathrm{mN}]$ \\
\hline Breakfast cereal & 80 \\
Coffee beans & 30 \\
Rice & 80 \\
Flour & 20 \\
\hline
\end{tabular}

2. Put the container on the hands manually to contact the edges of the container with the palms.

3. Start controlling the grasping force without moving the arm.

4. Move both arms until the target rotation angle is reached.

5. Move back to the initial positions.

For each container, we define the initial positions by giving grasping points. One grasping point is set to the center of the going-down side of the container. The other grasping point is set to the bottom that is near to the going-up side.

\section{Experiments}

\subsection{Pouring with Different Contents}

In this experiment, we use the same container (a thin aluminium bag) that is filled with one type of the contents as shown in Figure 8 . The purpose is to evaluate the effect of the type of contents. Ordered by size, there are coffee beans, cereal, a grains of rice, and flour powder. We set the target normal force as shown in Table 1. We choose the value so that the robot keeps the grasping in the end of tilting.

Figure 9 shows the robot pouring actually. As shown in this figure, the robot successfully poured even if the type of contents is different. Interaction among coffee beans is smoother since the size is big and the density is small. Thus, coffee beans are easily poured. Conversely, interaction among flour powder is sticky since the size is small. Flour suddenly starts being poured with a blob.

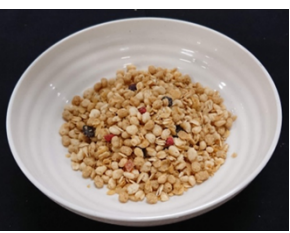

(a) Breakfast cereal

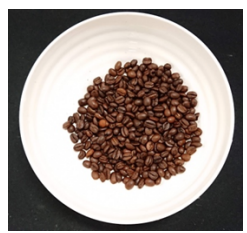

(b) Coffee beans

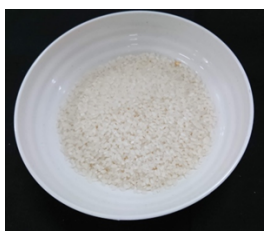

(c) Rice

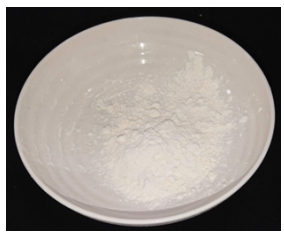

(d) Flour

Fig. 8. Target contents 

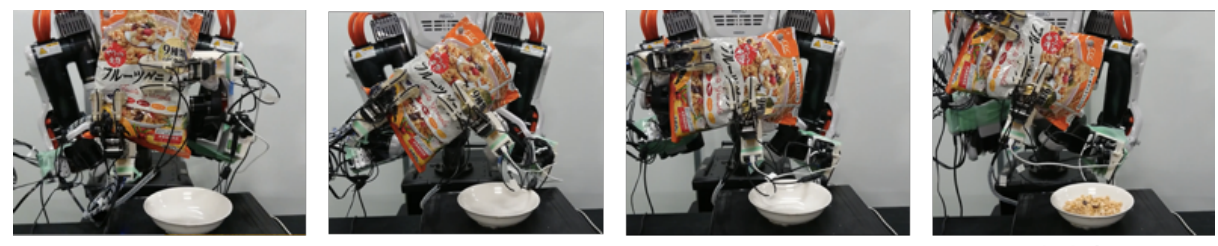

Start

(a) Breakfast cereal

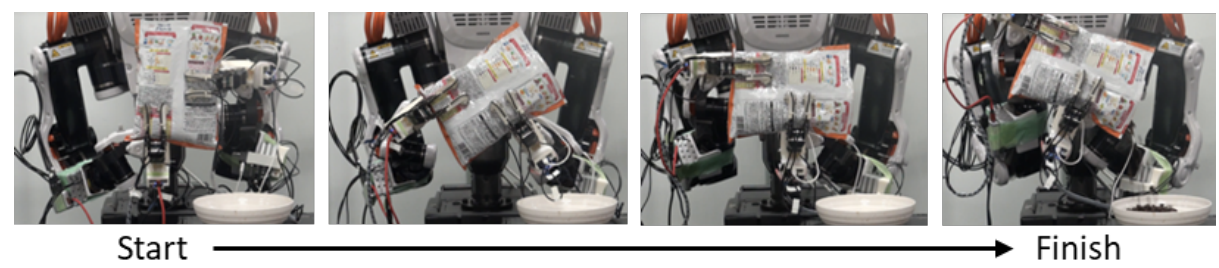

(b) Coffee beans
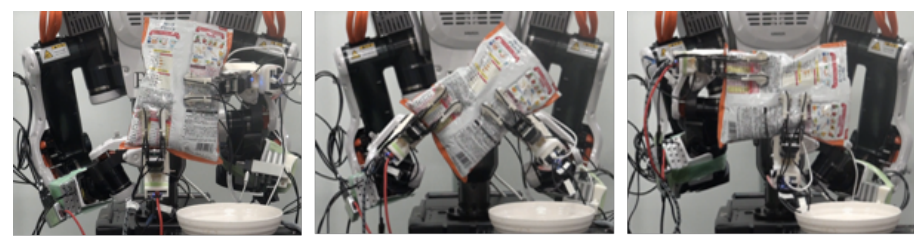

Start

(c) Rice

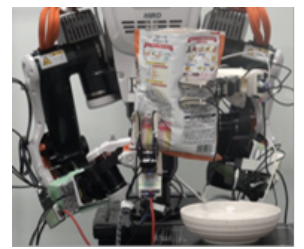

Start
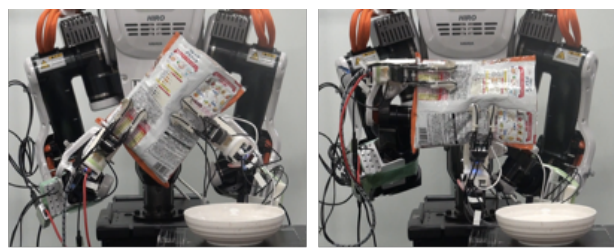

(1)

(d) Flour

Fig. 9. Experiment where a robot pours different contents from the same thin aluminium bag

\subsection{Pouring with Different Contents and Containers}

In this experiment, we use various types of contents and containers as shown in Figure 10. Except for rice in a plastic bag, Ziploc, we use commercial products in a grocery store. The targets are cereal in a thin-aluminum bag, coffee beans in a retort bag, rice in a Ziploc, and flour in a plastic bag. Table 2 shows the target normal forces.

Figure 11 shows the robot actual pouring. Though the deformation was different from each target, the robot successfully poured in all targets. We conclude 
Table 2. Grasping force for each product

\begin{tabular}{lr}
\hline Material & Force $[\mathrm{mN}]$ \\
\hline Breakfast cereal in a thin aluminum bag & 80 \\
Coffee beans in a retort pouch & 30 \\
Rice in Ziploc & 150 \\
Flour in a plastic bag & 50 \\
\hline
\end{tabular}

that the proposed system can handle various types of contents and containers by adjusting the normal force and grasping points.

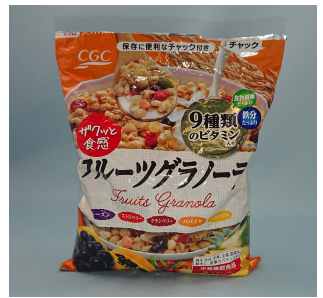

(a) Breakfast cereal

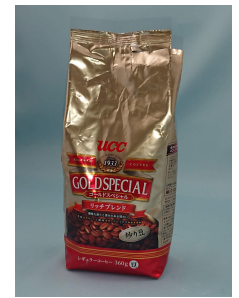

(b) Coffee beans

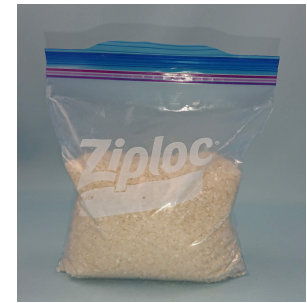

(c) Rice

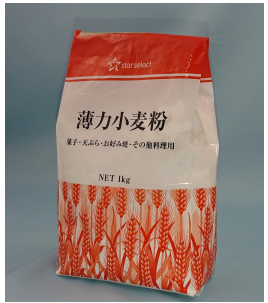

(d) Flour

Fig. 10. Target contents and containers

\subsection{Evaluation in Success Rate of Pouring}

We further evaluate the performance of the proposed system using the same situation of the experiment in Section 5.2. In this subsection, we evaluate the system using the successful rate of pouring. The robot pours each type of contents twenty times. We define three failure cases of pouring as follows:

- Case 1: Drop the container due to the weakness of the grasping force

- Case 2: Spill the contents outside of the target dish because the deformation of the container leads to a shift of the spout.

- Case 3: Prevent pouring the contents by too firmly grasping the container, even though the tilt angle of the container is big enough for pouring.

We consider the pouring as a success if the result is different than the three failure cases. We do not consider the amount of contents to be poured.

Table 3 shows the success rate: $20 / 20$ with cereal, 19/20 with coffee beans, $18 / 20$ with rice, and 19/20 with flour. Failure case 3 happens with coffee beans and flour. Failure cases 2 and 3 happen with rice. Figure 12 shows Failure case 2 with rice: the rice is spilled outside because the container is excessively deformed and then the spout moves.

Note that the state of the contents is slightly different in each trial. Though the grasping force is controlled, the arms are moved in a feed-forward manner. To increase the success rate, it is necessary to adjust the motion or abort pouring by quickly returning to the initial position considering the condition of pouring. 


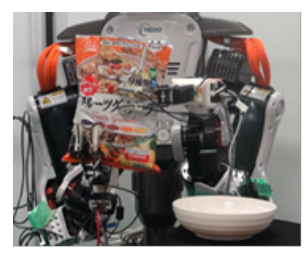

Start

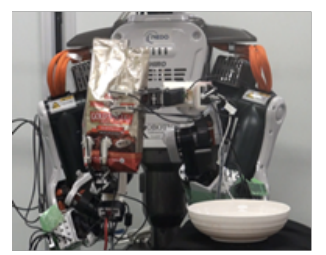

Start

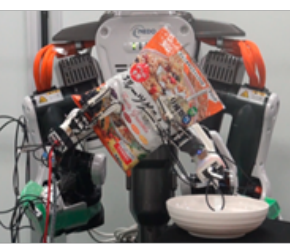

(a) Breakfast cereal
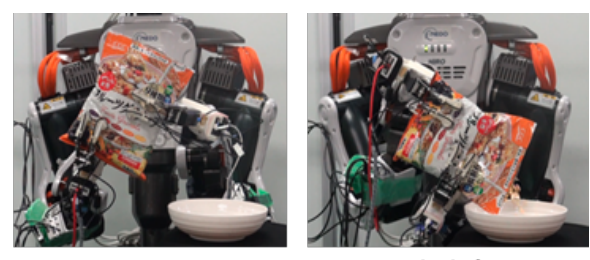

Finish
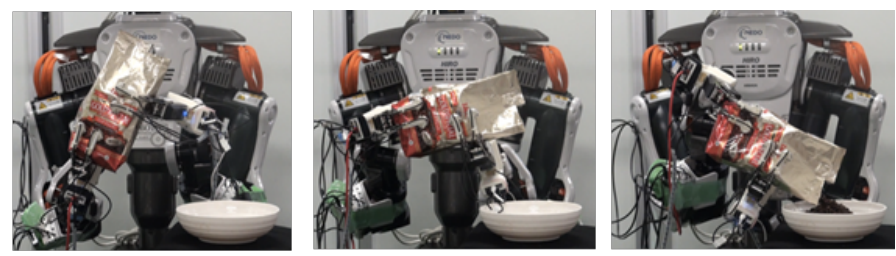

Finish

(b) Coffee beans

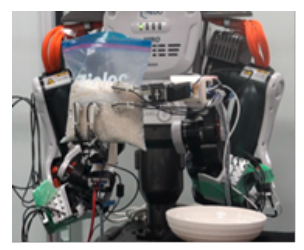

Start

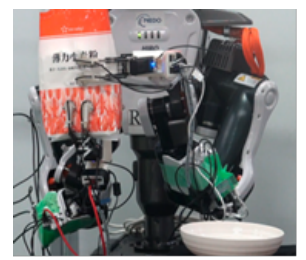

Start

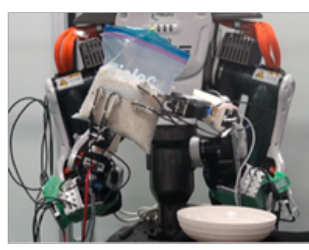

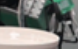
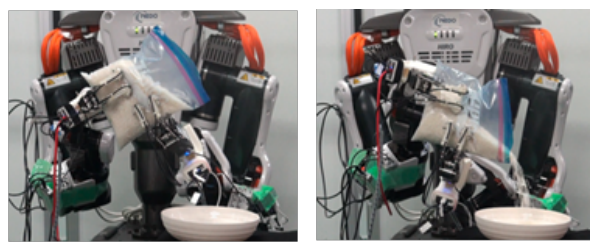

Finish

(c) Rice
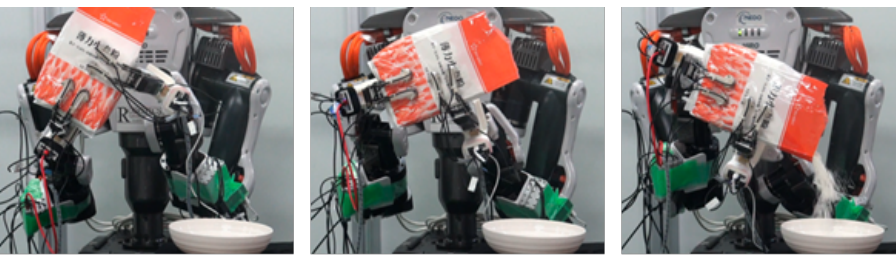

Finish

(d) Flour

Fig. 11. Experiment where a robot pours different contents from different containers Table 3. Success rate

\begin{tabular}{lrr}
\hline \multicolumn{3}{l}{ Material name Success rate [\%] } \\
\hline Cereal & 100 & $(=20 / 20)$ \\
Coffee beans & 95 & $(=19 / 20)$ \\
Rice & 90 & $(=18 / 20)$ \\
Flour & 95 & $(=19 / 20)$ \\
\hline
\end{tabular}




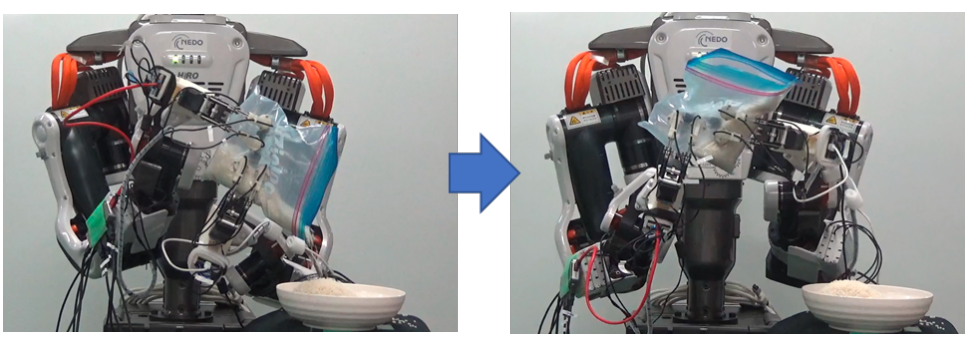

Fig. 12. Deform a container including rice

\subsection{Evaluation by the Amount of Deformation}

Our strategy is to reduce the deformation during pouring motion. In this subsection, we evaluate the amount of the deformation by measuring it. We compare the proposed method with the single arm case. We generate the motion of the single arm by removing the arm of the lifting hand.

To measure the deformation, we use a motion capture system (Figure 13), and we use Flex 3 cameras manufactured by OptiTrack Inc. We put eight cameras on the vertices of the rectangular frame. The directions of the cameras are adjusted as seeing the center of the frame. We attach the markers on the surface of the plastic container including rice. We chose this target since this container is more easily deformed than the others.

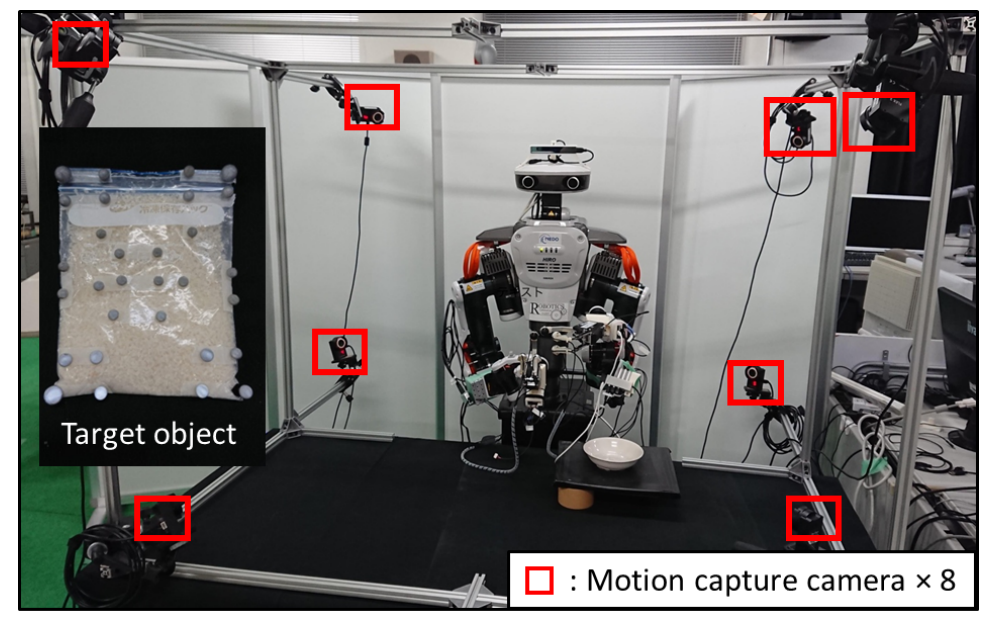

Fig. 13. Motion capture system to measure the deformation of the container during pouring motion

We measure the deformation in both pouring methods ten times. Figure 14 shows the example of the deformation in both methods. Figure 15 shows the surface of the container, which is estimated by fitting a cubic polynomial. After the pouring motion, the robot moves to the initial position and the displacement 
is evaluated in the initial position. Blue and red surfaces represent the surfaces before and after pouring. As can be seen, the surface with a single arm is more deformed than that of both arms.

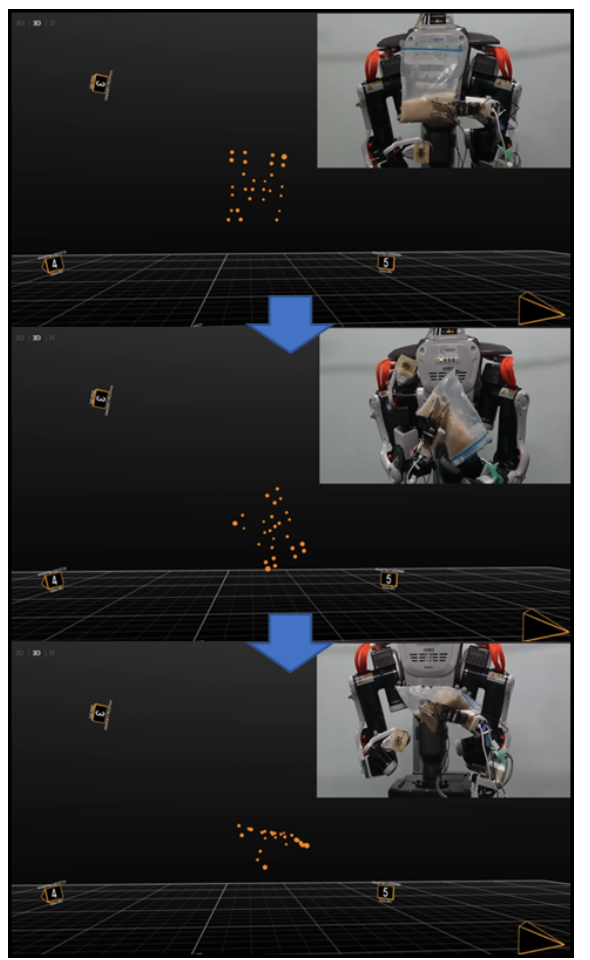

(a) single arm

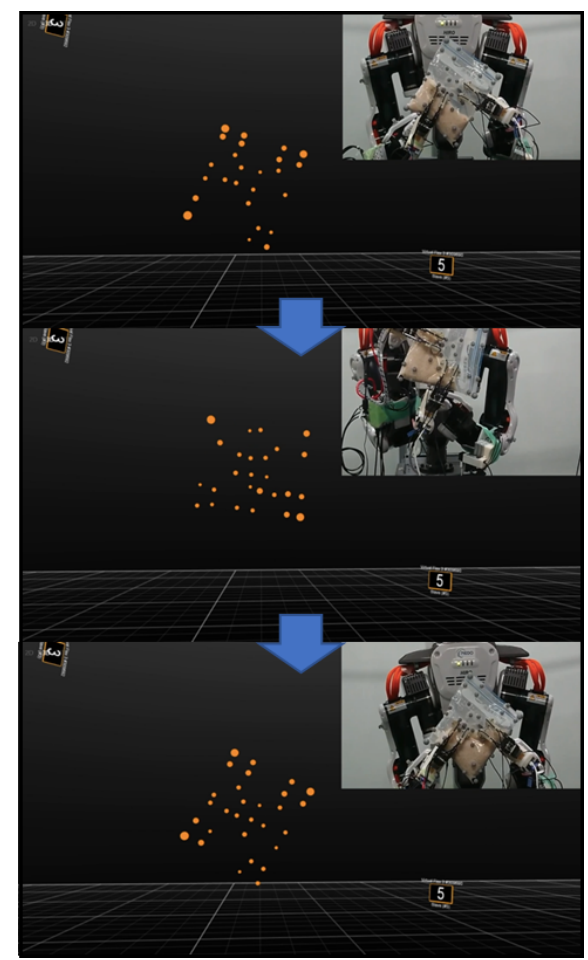

(b) dual arm

Fig. 14. Measured deformation of a plastic bag with a single-arm and dual-arm pouring

For quantitative evaluation, we calculate the displacement of the MoCap markers before and after the pouring motion. Table 4 shows the average of displacements of all markers. To calculate the average of all trials, we remove the maximum and minimum values from the samples to reduce the effect of outliers. The amount of deformation in dual-arm pouring is about $66 \%$ smaller than single-arm pouring.

\subsection{Investigate the Amount of Pouring}

We investigate the proposed system using the amount of pouring. The proposed system controls the motion in a feed-forward manner and the grasping force in a feedback manner. The amount of pouring depends on types of contents and containers. Even if we initialize contents and containers in a similar way, there is a possibility that the amount is slightly different. 


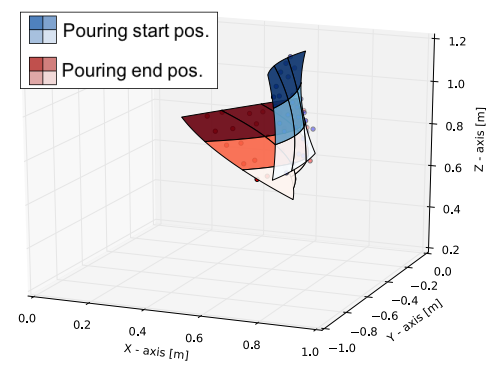

(a) Single arm

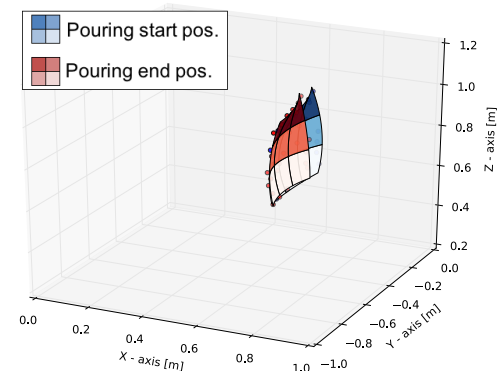

(b) Dual arm

Fig. 15. Deformation amount of the deformable container during the pouring motion Table 4. Results of deformation amount

\begin{tabular}{ccr}
\hline Trial num. Single arm $[\mathrm{mm}]$ & Dual arm $[\mathrm{mm}]$ \\
\hline 1 & 112 & 79 \\
2 & 138 & 73 \\
3 & 152 & 40 \\
4 & 150 & 83 \\
5 & 134 & 62 \\
6 & 203 & 43 \\
7 & 147 & 46 \\
8 & 219 & 33 \\
9 & 148 & 18 \\
10 & 108 & 49 \\
\hline Average & 158 & 53 \\
\hline
\end{tabular}

We need to compare the amount evenly in different targets. As shown in Figure 16, we control the amount of the filled contents using the ratio between the height $h_{i}$ of the contents and the height $h_{c}$ of the container. We set the ratio to 0.7 .

For pouring, the robot rotates a container until 120 degrees, as shown in Figure 17. We measure the amount of pouring using the height $h_{p}$ of the contents when placing the container upright. We define the pouring rate $P_{R}$ as

$$
P_{R}=\frac{h_{i}-h_{p}}{h_{i}} .
$$

As the rate becomes higher, the more contents are poured.

Figure 18 shows the rate of all the ten trials done for each content. Tables 5 to 8 show the maximum, minimum, and the average of the rate in the four products. The average with coffee beans is the highest while with flour is the lowest. The variance of the rates with coffee beans is larger; in some trials the robot poured almost all the beans, but in other trials the robot poured half of the beans. 


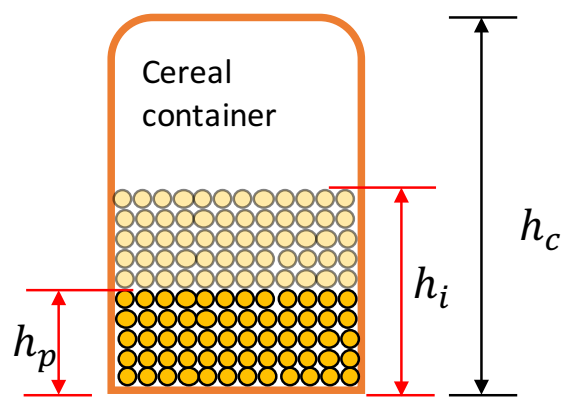

Fig. 16. Evaluation of the amount of contents

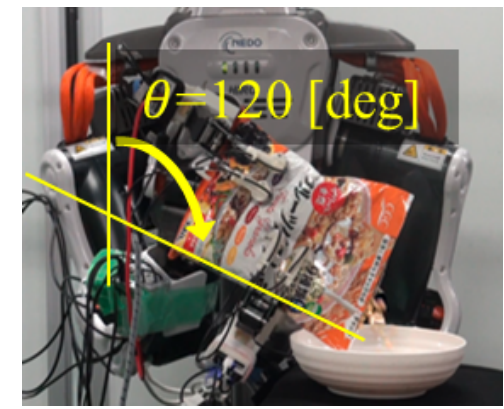

Fig. 17. Rotation angle of pouring motion

Table 5. Experimental parameters and results of breakfast cereal

\begin{tabular}{lrr}
\hline Parameter & Value \\
\hline Container height $h_{c}[\mathrm{~mm}]$ & 215 \\
Initial contents height $h_{i}[\mathrm{~mm}]$ & 150 \\
& Max & 70 \\
Pouring end contents height $h_{p}[\mathrm{~mm}]$ & Min & 103 \\
& Average & 87 \\
& Max & 53 \\
Pouring value rate [\%] & Min & 32 \\
& Average & 42 \\
\hline
\end{tabular}

Table 6. Experimental parameters and results of coffee beans

\begin{tabular}{lr}
\hline Parameter & Value \\
\hline Container height $h_{c}[\mathrm{~mm}]$ & 230 \\
Initial contents height $h_{i}[\mathrm{~mm}]$ & 160 \\
& Max 3 \\
Pouring end contents height $h_{p}[\mathrm{~mm}]$ & Min 80 \\
& Average 43 \\
& Max 98 \\
Pouring value rate [\%] & Min 50 \\
& Average 73 \\
\hline
\end{tabular}




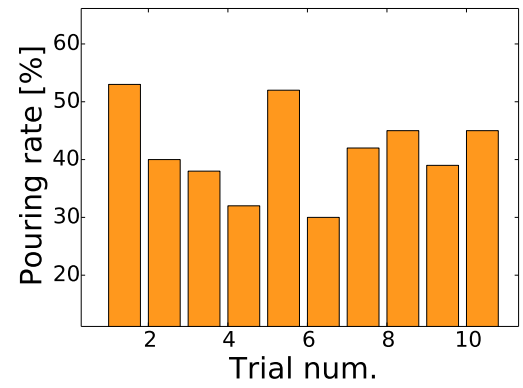

(a) Breakfast cereal

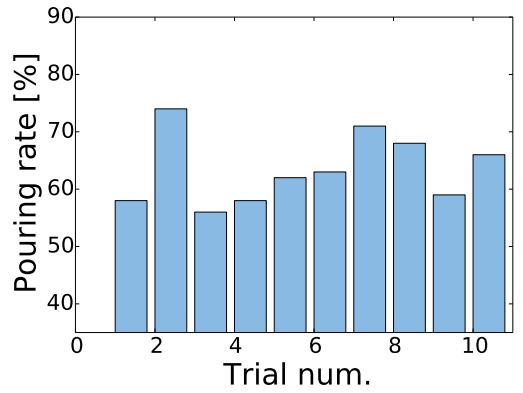

(c) Rice

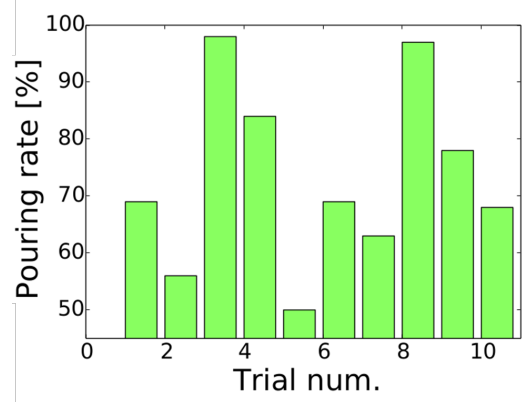

(b) Coffee beans

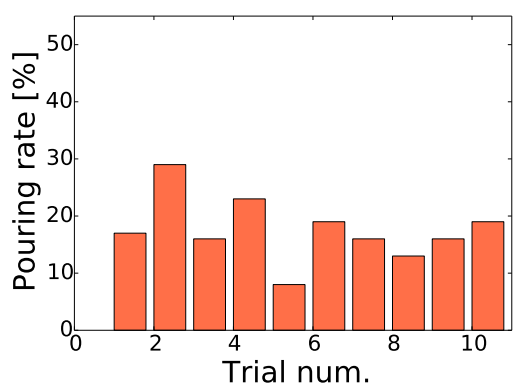

(d) Flour

Fig. 18. Pouring rate per trial

Table 7. Experimental parameters and results of rice

\begin{tabular}{lr}
\hline Parameter & Value \\
\hline Container height $h_{c}[\mathrm{~mm}]$ & 180 \\
Initial contents height $h_{i}[\mathrm{~mm}]$ & 125 \\
& Max 22 \\
Pouring end contents height $h_{p}[\mathrm{~mm}]$ & Min 53 \\
& Average 46 \\
& Max 74 \\
Pouring value rate [\%] & Min 58 \\
& Average 63 \\
\hline
\end{tabular}

\subsection{Discussion}

Through the experiments, we proved that the proposed system poured various contents from various deformable containers. The robot successfully poured the contents from the commercial products. However, the robot could not pour the whole contents from the containers since the contents were stuck in the surface of the container. To solve this, we are considering to implement a motion to shake the container. 
Table 8. Experimental parameters and results of flour

\begin{tabular}{|c|c|}
\hline Parameter & Value \\
\hline Container height $h_{c}[\mathrm{~mm}]$ & 225 \\
\hline \multirow[t]{2}{*}{ Initial contents height $h_{i}[\mathrm{~mm}]$} & 155 \\
\hline & $\operatorname{Max} 110$ \\
\hline \multirow[t]{3}{*}{ Pouring end contents height $h_{p}[\mathrm{~mm}]$} & Min 143 \\
\hline & Average 127 \\
\hline & $\operatorname{Max} 29$ \\
\hline \multirow[t]{2}{*}{ Pouring value rate $[\%]$} & Min \\
\hline & Average 18 \\
\hline
\end{tabular}

Even though we carefully initialized the starting condition, the pouring rate was diverse. Especially, the difference between the minimum and the maximum was about $50 \%$. Controlling the amount of pouring is out of scope of this paper. It is necessary to adjust the rotation angle of the container and/or the grasping force to control the flow of the poured contents using visual feedback.

\section{Conclusion}

In this paper, we proposed a robot system for pouring various contents from a deformable container using dual-arm holding and tactile information. To construct the proposed system, we adopted the strategy to reduce the deformation of the container during the pouring motion. We carefully design the grasping strategy: one hand supports the going-down side of the deformable container and the other hand lifts up the bottom near the going-up side in the container. We control the minimum grasping force to reduce the deformation.

In the experiments, the robot system successfully poured four different types of contents. Furthermore, the system poured three actual commercial products and rice in a Ziploc. We used two indices to evaluate the proposed system. The first index is the amount of deformation of the container. We compared the proposed system to a single-arm pouring. The experiments verified that the proposed system reduced the deformation by $66 \%$ compared to a singlearm pouring. The second index is the success rate of pouring. The experiments verified that the rate was greater than $90 \%$.

In the future, we will search for optimal grasping points for pouring motion with various types of deformable containers. In addition, we are planning to clarify the relationship between dual-arm holding and the reduction of deformation, and construct a system that performs a robust pouring motion with a deformable container by adjusting the motion to control the flow of the pouring contents. 


\section{References}

1. Doumanoglou, A., Stria, J., Peleka, G., Mariolis, I., Petrík, V., Kargakos, A., Wagner, L., Hlaváč, V., Kim, T.K., Malassiotis, S.: Folding clothes autonomously: A complete pipeline. IEEE Transactions on Robotics 32(6), 1461-1478 (2016)

2. Gemici, M.C., Saxena, A.: Learning haptic representation for manipulating deformable food objects. In: Proceeding of the IEEE/RSJ International Conference on Intelligent Robots and Systems (IROS). pp. 638-645 (2014)

3. Kennedy, M., Queen, K., Thakur, D., Daniilidis, K., Kumar, V.: Precise dispensing of liquids using visual feedback. In: IEEE/RSJ International Conference on Intelligent Robots and Systems (IROS). pp. 1260-1266. IEEE (2017)

4. Kunze, L., Beetz, M.: Envisioning the qualitative effects of robot manipulation actions using simulation-based projections. Artificial Intelligence 247, 352-380 (2017)

5. Lopez-Guevara, T., Taylor, N.K., Gutmann, M.U., Ramamoorthy, S., Subr, K.: Adaptable pouring: Teaching robots not to spill using fast but approximate fluid simulation. In: Proceedings of the 1st Annual Conference on Robot Learning. pp. 77-86 (2017)

6. Misimi, E., Olofsson, A., Eilertsen, A., Øye, E.R., Mathiassen, J.R.: Robotic handling of compliant food objects by robust learning from demonstration. In: Proceeding of the IEEE/RSJ International Conference on Intelligent Robots and Systems (IROS). pp. 6972-6979 (2018)

7. Mottaghi, R., Schenck, C., Fox, D., Farhadi, A.: See the glass half full: Reasoning about liquid containers, their volume and content. In: Proceeding of the IEEE International Conference on Computer Vision (ICCV). pp. 1871-1880 (2017)

8. Nadon, F., Valencia, A.J., Payeur, P.: Multi-modal sensing and robotic manipulation of non-rigid objects: A survey. Robotics 7(4), 74 (2018)

9. Pan, Z., Park, C., Manocha, D.: Robot motion planning for pouring liquids. In: International Conference on Automated Planning and Scheduling. pp. 518-526 (2016)

10. Paulius, D., Huang, Y., Milton, R., Buchanan, W., Sam, J., Sun, Y.: Functional object-oriented network for manipulation learning. In: IEEE/RSJ International Conference on Intelligent Robots and Systems (IROS). pp. 2655-2662 (2016)

11. Rodríguez, A.D., Corrales, J.A., Mezouar, Y., Lequievre, L., Jara, C., Torres, F.: Tactile control based on gaussian images and its applicationin bi-manual manipulation of deformable objects. Robotics and Autonomous Systems 94, 148-161 (2017)

12. Rozo, L., Jiménez, P., Torras, C.: Force-based robot learning of pouring skills using parametric hidden markov models. In: Proceedings of the 9th International Workshop on Robot Motion and Control (RoMoCo). pp. 227-232 (2013)

13. Sanchez, J., Corrales, J.A., Bouzgarrou, B.C.: Robotic manipulation and sensing of deformable objects in domestic and industrial applications: a survey. The International Journal of Robotics Research 37(7), 688-716 (2018)

14. Schenck, C., Fox, D.: Visual closed-loop control for pouring liquids. In: Proceeding of the IEEE International Conference on Robotics and Automation (ICRA). pp. 2629-2636 (2017)

15. Schenck, C., Fox, D.: Perceiving and reasoning about liquids using fully convolutional networks. The International Journal of Robotics Research 37(4-5), 452-471 (2018)

16. Tamosiunaite, M., Nemec, B., Ude, A., Wörgötter, F.: Learning to pour with a robot arm combining goal and shape learning for dynamic movement primitives. Robotics and Autonomous Systems 59(11), 910-922 (2011) 
17. Terashima, K., Yano, K., Sugimoto, Y., Watanabe, M.: Position control of ladle tip and sloshing suppression during tilting motion in automatic pouring machine 34(18), 229-234 (2001)

18. Tsuchiya, Y., Kiyokawa, T., Ricardez, G.A.G., Takamatsu, J., Ogasawara, T.: Pouring from deformable containers using dual-arm manipulation and tactile sensing. In: IEEE International Conference on Robotic Computing. pp. 357-362 (2019)

19. Tsuji, T., Noda, Y.: High-precision pouring control using online model parameters identification in automatic pouring robot with cylindrical ladle. In: IEEE International Conference on Systems Man and Cybernetics (SMC). pp. 2563-2568. IEEE (2014)

20. Yamaguchi, A., Atkeson, C.G.: Stereo vision of liquid and particle flow for robot pouring. In: Proceeding of the IEEE-RAS International Conference on Humanoid Robots (HUMANOIDS). pp. 1173-1180 (2016)

21. Yamaguchi, A., Atkeson, C.G., Niekum, S., Ogasawara, T.: Learning pouring skills from demonstration and practice. In: Proceeding of the IEEE-RAS International Conference on Humanoid Robots (HUMANOIDS). pp. 908-915 (2014)

22. Yamaguchi, A., Atkeson, C.G., Niekum, S., Ogasawara, T.: Learning skills from human demonstrations: Initial attempt on a pouring task. In: Proceedings of the 32nd Annual Conference of the Robotics Society of Japan (RSJ). pp. 1I3-04 (2014)

23. Yamaguchi, A., Atkeson, C.G., Ogasawara, T.: Pouring skills with planning and learning modeled from human demonstrations. International Journal of Humanoid Robotics 12(03) (2015)

24. Yang, P.C., Sasaki, K., Suzuki, K., Kase, K., Sugano, S., Ogata, T.: Repeatable folding task by humanoid robot worker using deep learning. IEEE Robotics and Automation Letters 2(2), 397-403 (2017) 